\title{
Effekte einer Stent-Implantation bei Venenerkrankungen der unteren Extremitäten
}

Mabud TS et al. Lower Extremity Venous Stent Placement: A Large Retrospective Single-Center Analysis. J Vasc Interv Radiol 2020; 31 : 251-259

Fragestellung der retrospektiven Studie um Mabud et al. war zum einen die Sicherheit und das kurz- und langfristige Behandlungsergebnis venöser Stents und zum zweiten die Charakterisierung der Veränderungen der venösen Durchmesser durch die Stent-Implantation bei sowohl thrombotischen als auch nichtthrombotischen Venenerkrankungen der unteren Extremitäten.

Die herangezogene Datenbank aller Patienten eines gefäßchirurgischen Zentrums (Stanford, Kalifornien), welche zwischen 1996 und 2018 eine Stent-Im- plantation im Bereich der unteren Extremitäten erhalten hatten, ergab 1094 Implantationen bei 406 Patienten (172 Männer, 234 Frauen, medianes Lebensalter 48,9 Jahre (Interquartile Range (IQR) 36-62,1)) in 513 Fällen einschließlich iliokavaler Stents. Die Indikationen waren

- bei 9,4\% akute Thrombosen,

- bei $65,3 \%$ chronische Thrombosen und bei

- bei $25,3 \%$ nichtthrombotische Läsionen.

Die primäre Offenheitsrate, die Offenheitsrate nach zwischenzeitlich notwendigen endovaskulären unterstützenden Maßnahmen (primary assisted patency) und die sekundäre Offenheitsrate (bis zu einem permanenten Verschluss) der venösen Stents wurden nach einem, 3 und 5 Jahren ermittelt. Subgruppenanalysen und Cox-Regression wurden angewandt, um Risikofaktoren für Verschlüsse zu bestimmen. Die Venendurchmesser und Villalta-Scores vor und 12 Monate nach Implantation wurden verglichen, Komplikationen und Mortalitätsraten wurden berechnet.

Stent-Auswahl und -Größe unterschieden sich je nach Venensegment und Präferenz des Untersuchers. Im Allgemeinen wurde eine auf Erfahrung basierte Auswahl an 
Stent-Durchmessern angewandt, d. h. für die V. cava inferior ( $\mathrm{VCl}$ ) 20-24 mm, für die $V$. iliaca communis $12-16 \mathrm{~mm}$, für die V. iliaca externa $12-14 \mathrm{~mm}$ und $10-$ 12 mm für Gefäße unterhalb des Ligamentums inguinale. Die Art des antikoagulatorischen Regimes richtete sich nach den Leitlinien der American Academy of Chest Physicians für tiefe Beinvenenthrombosen (nur Enoxaparin, Enoxaparin überbrückend zu Warfarin oder zu Rivaroxaban oder zu einem anderen Antikoagulans wie Fondaparinux oder Dabigatran).

Die primäre, primary assisted und sekundäre Offenheitsrate nach 5 Jahren betrugen 57,3\%, 77,2\% und 80,9\% (nach KaplanMeier) respektive 78,6\%, 90,3\% und 92,8\% (summary statistics). Die mediane Nachbeobachtungszeit lag bei 199 Tagen (IQR 35,2-712,0). Die Offenheitsraten in der Subgruppe mit $\geq 5$ Jahren Nachbeobachtung ( $n=46$; Mittelwert \pm Standardabweichung (SD) 9,1 $\pm 3,4$ Jahre) waren nahezu identisch mit den Raten der Studienteilnehmer zum Zeitpunkt von 5 Jahren Nachbeobachtung. Patienten mit einer StentImplantation in die V. cava inferior (Hazard
Ratio (HR) 2,11; $95 \%$-Konfidenzintervall (KI) 1,38-3,22; $\mathrm{p}<0,0001)$ oder mit einer akuten Thrombose (HR 3,65; $95 \%$-KI 1,747,64; $p<0,0001$ ) während der Index-Prozedur hatten ein signifikant erhöhtes Risiko, ihre primäre Durchgängigkeit wieder zu verlieren. Thrombophiliestatus, Beinseite und das angewandte antikoagulatorische Regime waren statistisch nicht signifikant assoziiert mit einem Verschluss.

Die Venendurchmesser waren nach StentImplantation signifikant größer. Die Messungen waren dabei im Mittel 67 Tage vor und 91 Tage nach dem Index-Eingriff erfolgt.

Es gab keinen Fall von Stent-Fraktur, -Migration oder strukturellen Deformierungen, obwohl die verwendeten Stents nicht speziell entworfen und von der US Food and Drug Administration für venöse Interventionen nicht geprüft und zugelassen sind. Bei Patienten mit chronischer tiefer Beinvenenthrombose verringerten sich die Punktwerte im Villalta-Score nach Stent-Implantation (von 15,7 $\pm 8,6$ auf $7,4 \pm 6,5 ; p<0,0001)$. Die perioperative
Sterblichkeit lag unter $1 \%$ und die Rate schwerer perioperativer Komplikationen betrug 3,7\%. Keiner der 4 Todesfälle ließ sich mit der Stent-Prozedur in Zusammenhang bringen.

\section{FAZIT}

Eine kavo-ilio-femorale Stent-Implantation bei venöser Verschlusskrankheit bewirkt eine Verbesserung des Schweregrades der Erkrankung (VillaltaScore), eine Zunahme des Durchmessers der behandelten Venen und zufriedenstellende Offenheitsraten im Langzeitverlauf. Eine ausreichende radiale Spannkraft zur Aufrechterhaltung der nominalen Durchmesser der Stents sollte bei einer zukünftigen Entwicklung speziell für die biomechanischen Verhältnisse bei Venen entworfener Implantate berücksichtigt werden.

Dr. Birgit Gappa, Penzberg 